\title{
ESTUDO SOBRE A INFLUÊNCIA DE VARIÁVEIS METEOROLÓGICAS EM INTERNAÇÕES HOSPITALARES EM MACEIÓ-AL, DURANTE O PERÍODO 1998 A 2006
}

\author{
DANIEL DOS SANTOS, MANOEL DA ROCHA TOLEDO FILHO
}

\author{
Universidade Federal de Alagoas (UFAL), Instituto de Ciências Atmosféricas, Maceió, AL, Brasil \\ daniel.meteorologia@yahoo.com.br, mrtoledofilho@yahoo.com.br
}

Recebido Julho de 2011 - Aceito Janeiro de 2013

\begin{abstract}
RESUMO
Foram utilizados dados de insolação, pressão atmosférica, velocidade do vento, temperatura do ar, umidade relativa do ar e precipitação pluviométrica de valores médios mensais do período de 1998 a 2006, de Maceió, objetivando identificar a influência destas variáveis meteorológicas nas internações hospitalares por asma, leptospirose, insuficiência cardíaca, esquistossomose e bronquite aguda. Determinaram-se os coeficientes de internações mensais em hospitais públicos de Maceió, relacionando-os com as variáveis estudadas no período estudado. Calculou-se o coeficiente de correlação de Pearson ( $\mathrm{r}$ ) e coeficiente de determinação $\left(\mathrm{r}^{2}\right)$ das variáveis meteorológicas, para saber, o máximo que estes explicam as internações estudadas. $\mathrm{O}$ resultado sugere que houve influência das variáveis meteorológicas nos casos de internações por asma, com $\mathrm{r}^{2}(\%)$ de $0 \%$ a $9 \%$, bronquite o $\mathrm{r}^{2}$ foi de $29 \%$ a $55 \%$, para esquistossomose $15 \%$ a $45 \%$, insuficiência cardíaca $0 \%$ a $11 \%$ e leptospirose $14 \%$ a $79 \%$. Palavras-chave: variáveis meteorológicas, correlação estatística, doenças.
\end{abstract}

\begin{abstract}
STUDY OF METEOROLOGICAL VARIABLE INFLUENCES ON HOSPITAL ADMISSIONS IN MACEIO-AL DURING THE 1998-2006 PERIOD

Monthly average values of insolation, atmospheric pressure, wind speed, air temperature, relative humidity and pluviometric precipitation in Maceió from 1998 to 2006 were used to identify their influence in hospitalizations due to asthma, leptospirosis, cardiac insufficiency, schistosomiasis and chronic bronchitis. Coefficients of monthly admissions in public hospitals of Maceió were determined and then correlated to the variables of interest. The Pearson (r) and determination (r2) coefficients of the meteorological variables were calculated to identify the extent of their influence over hospital admissions. The results suggest the confirmation of the author's hypothesis about the influence of weather conditions on hospitalizations due to asthma $\left(\mathrm{r}^{2}\right)$ from $0 \%$ to $9 \%$, bronchitis $\left(\mathrm{r}^{2}\right)$ from $29 \%$ to $55 \%$, schistosomiasis $(15 \%$ to $45 \%)$, cardiac insufficiency ( $0 \%$ to $11 \%)$ and leptospirosis (14\% to $79 \%)$.
\end{abstract}

Keywords: meteorological variables, statistical correlation, diseases.

\section{INTRODUÇÃO}

Os problemas de saúde pública por doenças infecciosas são notórios em algumas regiões do Brasil. Em Maceió, Alagoas, esse problema se agrava por motivo da falta de infra-estrutura principalmente em regiões de risco. Sabem-se, pela literatura, que algumas doenças infecciosas são sensíveis às condições climáticas ocorridas em uma região, geralmente citando o inverno ou verão.

A Biometeorologia busca estudar essa relação e com seus resultados, encontrar informações que tentem minimizar os efeitos dessas doenças, indicando os períodos de suas maiores incidências. A hipótese principal desta pesquisa é que em Maceió algumas doenças infecciosas estão relacionadas com os elementos meteorológicos e climatológicos, ou seja, seus agravos notificáveis respondem em algumas situações climáticas específicas.

Esta pesquisa consistiu em determinar quais variáveis meteorológicas são capazes de influenciar casos de internamentos hospitalares de algumas doenças infecciosas. Neste trabalho foram utilizados dados de temperatura do ar, umidade relativa do ar, precipitação pluviométrica, insolação, pressão atmosférica e 
velocidade do vento. Dentre essas seis variáveis meteorológicas, três delas normalmente não são utilizadas em trabalhos deste tipo, como a insolação, pressão atmosférica e velocidade do vento.

O objetivo geral foi de estudar as correlações estatísticas entre variáveis meteorológicas e internações hospitalares de alguns pacientes em Maceió-AL, durante o período 1998 a 2006.

Durante certas condições meteorológicas, algumas pessoas podem queixar-se de problemas de mal-estar ou mesmo de doença. Percebe-se que idosos, crianças e jovens são mais susceptíveis as mudanças meteorológicas. Já se provou estatisticamente, que os eventos meteorológicos podem ter uma grande influência nos humanos e nos animais. Durante a onda de calor do verão de 2003 a taxa de mortalidade aumentou algumas vezes o seu valor habitual nos países da Europa (Uherek, 2005).

A Asma é uma doença que afeta cerca de $10 \%$ da população mundial. Conhecida como Asma Brônquica ou Bronquite Asmática, ela é uma doença crônica caracterizada pela inflamação dos pulmões, e também por causa alérgica, que leva a uma constrição de brônquios e bronquíolos, de ambos os pulmões, ocasionando dificuldade de respirar. Em 1996, no Brasil, a asma foi a $4^{\mathrm{a}}$ causa de internação nos hospitais do Sistema Único de Saúde e é responsável por uma média de 2.000 óbitos/ano, aproximadamente $70 \%$ destes durante a hospitalização dos doentes (Bettencourt, 2002). Os sintomas podem aparecer a qualquer momento do dia, mas tendem a predominar pela manhã ou à noite. Caracteristicamente, os sintomas aparecem de forma cíclica, com períodos de piora. Dentre os sinais e sintomas principais, estão: tosse, que pode ou não estar acompanhada de alguma expectoração (catarro), falta de ar, chiado no peito (sibilância), dor ou "aperto" no peito (Lança, 2001).

A Bronquite aguda é uma inflamação da árvore brônquica, geralmente associada com uma infecção respiratória generalizada. A árvore brônquica é composta por tubos (brônquios) que carregam o ar para dentro dos pulmões. Quando esses tubos estão com alguma infecção ficam edemaciados e produzem muco espesso. Isto pode tornar a respiração difícil. Ela é uma doença respiratória aguda, com tosse intensa e prolongada, que persiste por mais tempo após o desaparecimento dos outros sintomas respiratórios. A doença pode tornar a árvore brônquica mais sensível ao ar frio e a poluentes como a fumaça do cigarro, fazendo com que o indivíduo tenha tosse intensa quando se defronta com tais situações. Esta é uma doença que ocorre mais comumente durante os meses de inverno. Ela é quase sempre causada por viroses que atacam a mucosa dos brônquios, causando a infecção. Na maioria das vezes, as mesmas viroses que causam resfriados, causam a bronquite aguda. As viroses que causam bronquite aguda espalham-se pelo ar. Se o indivíduo sadio respirar o ar contaminado por vírus deixado pela tosse de um doente, poderá adquirir a doença. Isso também poderá acontecer ao tocar com a mão uma superfície contaminada por vírus e, após, leva-la até o nariz ou a boca. A superfície contaminada pode ser a mão de um indivíduo doente ou um objeto tocado por ele. A manifestação mais proeminente da bronquite aguda é a tosse (Lança, 2001).

A Esquistossomose é uma infecção causada por verme parasita da classe Trematoda, que ocorre em diversas partes do mundo de forma não controlada. No nosso país a esquistossomose é causada pelo Schistossoma mansoni, que é um dos cinco tipos que existe no mundo. O principal hospedeiro e reservatório do parasita é o homem, sendo a partir de suas fezes e urina que os ovos são disseminados na natureza. Possui ainda um hospedeiro intermediário que são os caramujos, caracóis ou lesmas, onde os ovos passam a forma larvária. Esta última dispersa principalmente em águas não tratadas, como lagos, infecta o homem pela pele causando uma inflamação da mesma. Quando esta no homem, o parasita se desenvolve e se aloja nas veias do intestino e fígado, causando obstrução das mesmas, sendo esta a causa da maioria dos sintomas da doença que pode ser crônica e levar a morte. Os ovos eliminados pela urina e fezes dos homens contaminados evoluem para larvas na água, estas se alojam e desenvolvem em caramujos. Estes últimos liberam a larva adulta, que ao permanecer na água contaminam o homem. No sistema venoso humano os parasitas se desenvolvem até atingir de 1 a $2 \mathrm{~cm}$ de comprimento, se reproduzem e eliminam ovos. O desenvolvimento do parasita no homem leva aproximadamente 6 semanas (período de incubação), quando atinge a forma adulta e reprodutora já no seu habitat final, o sistema venoso. A liberação de ovos pelo homem pode permanecer por muitos anos. No momento da contaminação, pode ocorrer uma reação do tipo alérgica na pele com coceira e vermelhidão, desencadeada pela penetração do parasita. Esta reação ocorre aproximadamente 24 horas após a contaminação. Após 4 a 8 semanas surge quadro de febre, calafrios, dor de cabeça, dores abdominais, falta de apetite, náuseas, vômitos e tosse seca (Torelly, 2004).

O coração é um músculo formado por duas metades, à direita e a esquerda, quando uma dessas cavidades falha como bomba, não sendo capaz de enviar adiante todo o sangue que recebe, fala-se que há insuficiência cardíaca (IC), ela não é uma doença do coração por si só, mais também uma incapacidade do coração efetuar as suas funções de forma adequada como consequência de outras enfermidades, do próprio coração ou de outros órgãos. Existem dois tipos de insuficiência cardíaca, a Insuficiência Cardíaca Aguda (ICA) e a Insuficiência Cardíaca Congestiva (ICC). A Insuficiência Cardíaca Aguda é um acontecimento súbito, que ocorre devido a qualquer situação que torne o coração incapaz de uma ação eficaz. Geralmente a Insuficiência Cardíaca Aguda é consequente a um infarto do miocárdio, ou a uma arritmia severa do coração. Existem ainda 
as provocadas por doenças não cardíacas, um exemplo delas são a hemorragia severa, o traumatismo cerebral grave e o choque elétrico de alta voltagem. A Insuficiência Cardíaca Aguda é uma situação grave, exige tratamento médico emergencial, e mesmo assim é, muitas vezes, fatal. (Eye, 2001). Estudos prévios demonstraram que ocorrem mais episódios de infarto agudo do miocárdio (ataques cardíacos) na época do inverno e em determinados horários do dia. As mudanças na pressão barométrica mostram variações diárias e nas diversas estações do ano, e poderiam modular a ocorrência de eventos vasculares. Um novo estudo, publicado na revista American Journal of Cardiology, objetivou determinar se existe uma relação entre mudanças na pressão barométrica e a ocorrência de ataque cardíaco na região central do Texas entre 1993 e 1996. Foram identificados um total de 1.327 pacientes vítimas de ataque do coração e de 839 pacientes vítimas de acidente vascular cerebral. O estudo indicou que o outono e o inverno mostraram uma maior variabilidade nas leituras de pressão atmosféricas. Segundo os investigadores, um risco aumentado de ataque do coração, mas não de derrame cerebral, foi observado no dia que segue a uma queda na pressão atmosférica (Campos, 2005).

Leptospirose é uma doença infecciosa, uma zoonose, causada por uma série de bactérias, chamada leptospira. A forma mais grave da doença e com mais alta mortalidade é associada ao Leptospira icterohaemorrhagiae, chamada com mais frequentemente de doença de Weil. A infecção humana na maioria das vezes está associada ao contato com água, alimentos ou solo contaminados pela urina de animais portadores do leptospira. As bactérias são ingeridas ou entram em contato com a mucosa ou pele que apresentem solução de continuidade. Os animais classicamente lembrados são os roedores, mas bovinos, equinos, suínos, cães e vários animais selvagens são responsabilizados pela difusão da doença. A contaminação entre pessoas doentes é absolutamente rara (Oliveira, 2001).

Esta pesquisa visa identificar as variáveis meteorológicas que possuem maior influência sobre as doenças estudadas e verificar o grau dessa influência com ajuda de métodos estatísticos.

\section{MATERIAIS E MÉTODOS}

As informações meteorológicas foram fornecidas pelo Instituto Nacional de Meteorologia (INMET), referentes à estação de Maceió-AL $\left(09,67^{\circ} \mathrm{S}, 35.7^{\circ} \mathrm{W}, 64,5 \mathrm{~m}\right)$. Foram utilizados dados de precipitação pluviométrica (totais mensais), umidade relativa do ar, pressão atmosférica, insolação, velocidade média do vento e temperatura do ar durante o período de 1998 a 2006.

As informações relativas ao total de número de casos de internamentos hospitalares mensais das doenças ocorridas durante o período estudado foram obtidas pelo Ministério da Saúde, através do Sistema de Informações Hospitalares do SUS (DATASUS, 2009). Segundo o Ministério da Saúde, o SUS tem 6,1 mil hospitais credenciados, 45 mil unidades de atenção primária e 30,3 mil Equipes de Saúde da Família (ESF). O sistema realiza 2,8 bilhões de procedimentos ambulatoriais anuais, 19 mil transplantes, 236 mil cirurgias cardíacas, 9,7 milhões de procedimentos de quimioterapia e radioterapia e 11 milhões de internações (BRASIL, 2013).

Foram elaborados perfis das variáveis citadas acima para a análise da relação entre cada variável meteorológica e o número de internações hospitalares. Em seguida efetuou-se o cálculo dos coeficientes de correlação de Pearson (r), utilizando a metodologia transcrita em Oliveira (2004), que apresenta como variável x (variável independente), as variáveis meteorológicas e como variável y (variável dependente), os valores dos totais mensais do número de casos de internamento pelas doenças estudadas (Equação 1).

Coeficiente de determinação é o quadrado do coeficiente de correlação de Pearson e pode ser chamado simplesmente $\mathrm{r}^{2}$. É uma medida da proporção da variabilidade em uma variável que é explicada pela variabilidade da outra. É pouco comum que ter uma correlação perfeita $\left(\mathrm{r}^{2}=1\right)$ na prática, porque existem muitos fatores que determinam as relações entre variáveis na vida real (Shimakura, 2006).

$$
r=\frac{n \sum x y-\left(\sum x\right)\left(\sum y\right)}{\sqrt{n\left(\sum x^{2}\right)-\left(\sum x\right)^{2}} \sqrt{n}\left(\sum y^{2}\right)-\left(\sum y\right)^{2}}
$$

Onde: $r$, representa o coeficiente de correlação linear para uma amostra $e n$ o número de pares de dados presentes;

Para as análises das magnitudes das correlações, recorreu-se aos valores de $\mathrm{r}^{2}$ processados por Cavalcante (2003), conforme a Tabela 1.

\section{RESULTADOS E DISCUSSÕES}

A linha representativa das internações por asma possui inúmeras variações (Figura 1), ocorrendo os maiores casos de asma durante o período de setembro a novembro. Relacionando a temperatura do ar ao número de internamentos na cidade de Maceió, permite constatar uma relação inversa entre a linha que representa o número de casos de internações e a linha da temperatura do ar ao decorrer do ano. A temperatura do ar apresenta suas maiores magnitudes entre os meses de novembro a maio. Os meses de novembro registram elevados registros de internações (90,6 casos), notadamente o mês com alguns dos maiores valores de temperatura do ar $\left(25,6^{\circ} \mathrm{C}\right)$.

Verificando as relações entre as variáveis meteorológicas e casos de internações (Figura 2), pode notar-se, que entre as seis variáveis meteorológicas, a pressão atmosférica e a 
Tabela 01 - Classificação dos valores dos coeficientes de correlação de Pearson (r) e de determinação (r2).

\begin{tabular}{c|c|c}
\hline $\mathbf{r}$ & $\mathbf{r}^{\mathbf{2}}$ & Classificação \\
\hline 0 & 0 & Nula \\
\hline 0,00 ------| 0,30 & 0,00 ------ 0,09 & Fraca \\
\hline 0,30 -----| 0,60 & 0,09 ------ 0,36 & Média \\
\hline 0,60 ------| 0,90 & 0,36 ------| 0,81 & Forte \\
\hline 0,90 ------| 0,99 & 0,81 ------ 0,99 & Fortíssima \\
\hline 1 & 1 & Perfeita \\
\hline
\end{tabular}

Fonte: Cavalcante, (2003) temperatura do ar possuem uma maior correlação com os números de internações hospitalares por asma. Destaca-se também a correção com a umidade relativa do ar. Mostrando que quanto maior é a pressão atmosférica e a umidade relativa do ar, e menor a temperatura do ar, mais casos de internações hospitalares por asma ocorrem. Esses resultados estão de acordo com a revisão bibliográfica de Bibliomed (2008).

De acordo com a Tabela 1 e a Figura 3, pode-se notar que o coeficiente de determinação entre as seis variáveis meteorológicas, mostra que a pressão atmosférica e a temperatura do ar possuem os melhores resultados com aproximadamente $9 \%$ de relação com os casos de internações hospitalares por asma.

Os valores de internações por bronquite possuem grandes variações (Figura 4), ocorrendo os maiores casos de

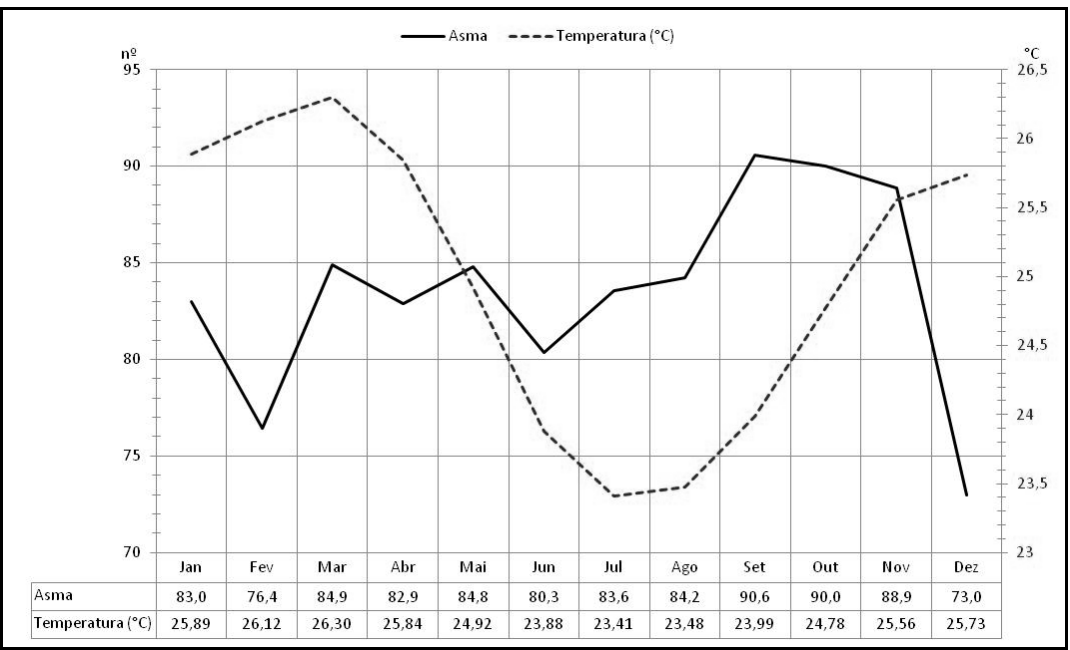

Figura 1 - Relação entre totais mensais dos números de internamentos hospitalares por asma na cidade de Maceió-AL e média da temperatura do ar.

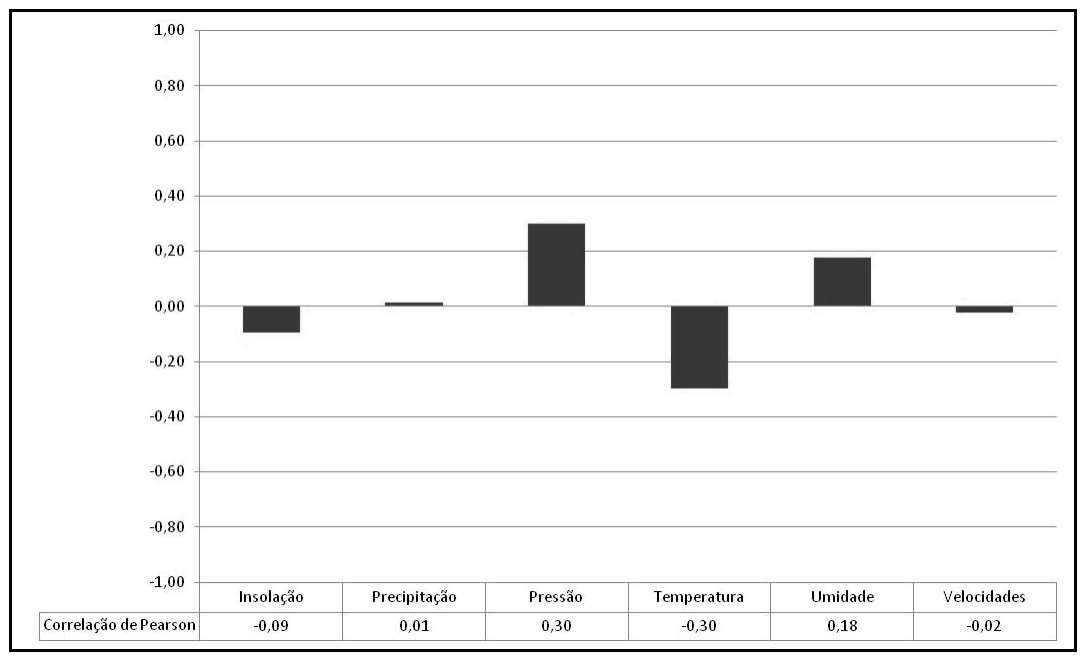

Figura 2 - Correlação de Pearson entre totais mensais dos números de internamentos hospitalares por asma na cidade de Maceió-AL e média de seis variáveis meteorológicas. 


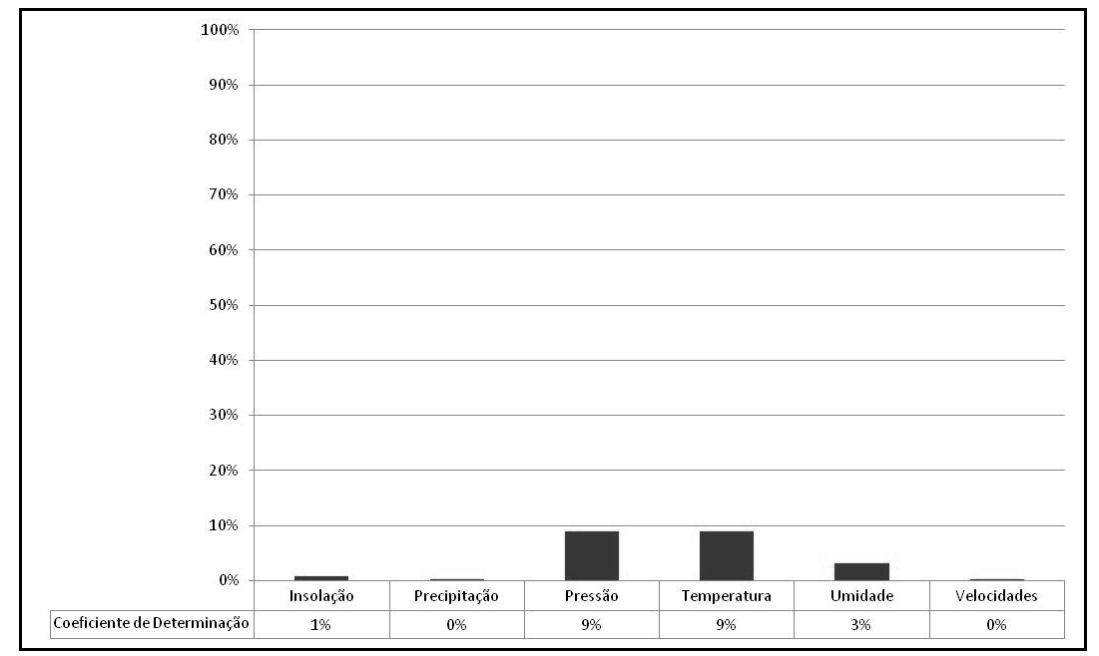

Figura 3 - Coeficiente de Determinação entre totais mensais dos números de internamentos hospitalares na cidade de Maceió-AL e média de seis variáveis meteorológicas.

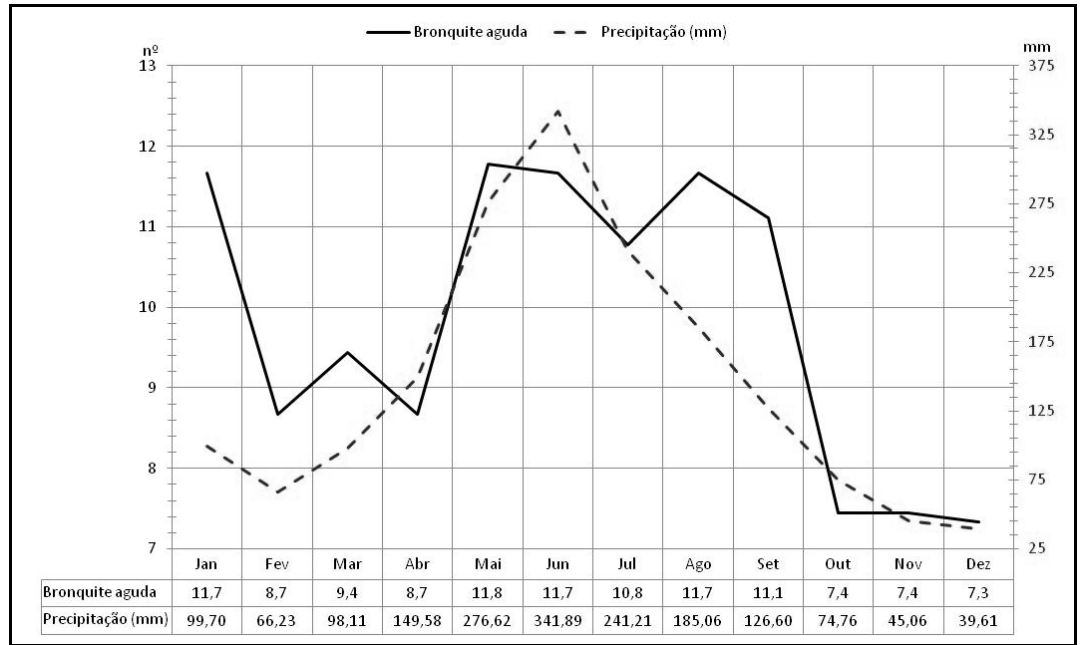

Figura 4 - Valores médios mensais dos números de internamentos hospitalares por bronquite aguda na cidade de Maceió-AL e média mensal da precipitação pluviométrica total.

internamento durante o período de maio a setembro e nos meses de Janeiro. Visualizando a linha da precipitação pluviométrica total, pode ser notado um grande aumento no meio do ano (maio a agosto). Relacionando a precipitação pluviométrica ao número de internamentos na cidade de Maceió, nota-se que o mês de dezembro teve um dos menores casos de internações hospitalares ( 7,3 casos), este mês coincidiu com o mês de menor precipitação pluviométrica $(39,61 \mathrm{~mm})$.

Utilizando a equação de Pearson, as variáveis meteorológicas e casos de internações, pode-se notar na Figura 5, que entre as seis variáveis meteorológicas, a pressão atmosférica e a insolação, precipitação pluviométrica, umidade relativa do ar e velocidade média do vento possuem correlação forte e as outras variáveis estudadas possuem correlação média com os números de internações hospitalares por bronquite aguda. Mostrando que quanto maior a precipitação pluviométrica, pressão do ar, umidade relativa do ar e menor a insolação, temperatura do ar e velocidade média do vento, poderá haver mais casos de internações hospitalares por bronquite aguda.

O coeficiente de determinação entre as seis variáveis meteorológicas (Figura 6), mostra que a precipitação pluviométrica e a umidade relativa do ar possuem os melhores resultados com $55 \%$ e $53 \%$ de relação com os casos de internações hospitalares por bronquite aguda.

A linha representativa da esquistossomose é descontínua e marcada por algumas rupturas (Figura 7). Destacando-se os meses de Janeiro e Dezembro por terem os menores valores (3,2 e 3,9 casos), e os meses de Fevereiro e agosto por possuírem os maiores casos de internações (6 e 6,8 casos). Observando a linha da velocidade média do vento, nota-se um primeiro período de queda de velocidade, e em seguida um aumento na velocidade média do vento ao longo do ano. A velocidade do 


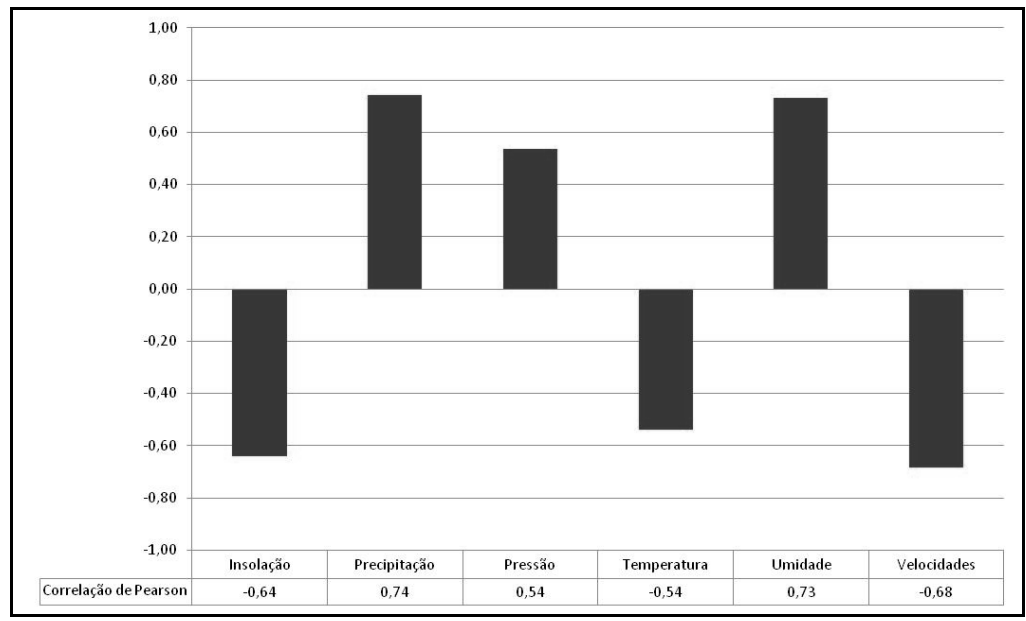

Figura 5 - Correlação de Pearson entre totais mensais dos números de internamentos hospitalares por bronquite aguda na cidade de Maceió-AL e média de seis variáveis meteorológicas.

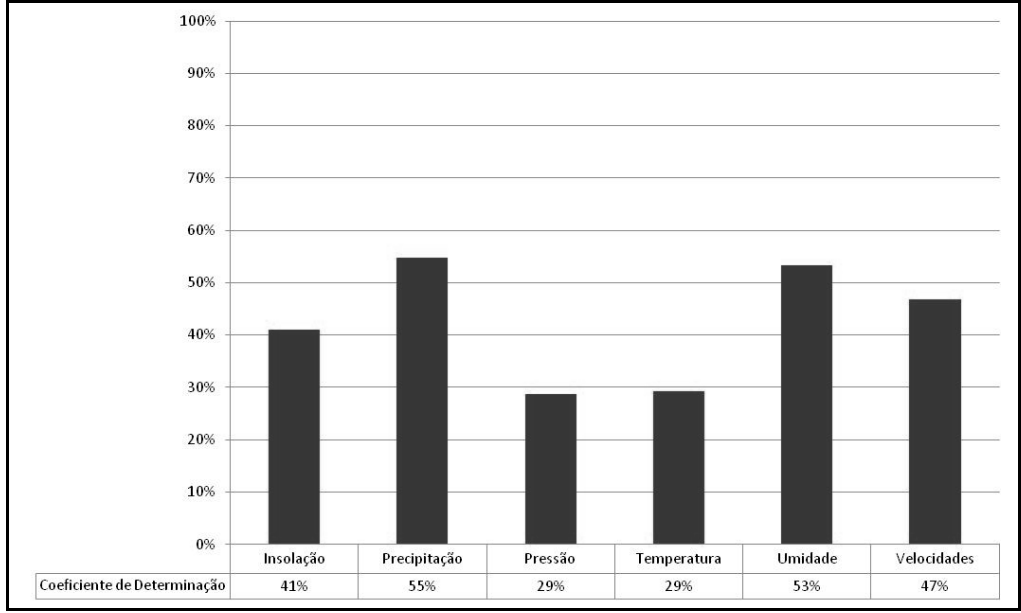

Figura 6 - Coeficiente de Determinação entre totais mensais dos números de internamentos hospitalares por bronquite aguda na cidade de MaceióAL e média de seis variáveis meteorológicas.

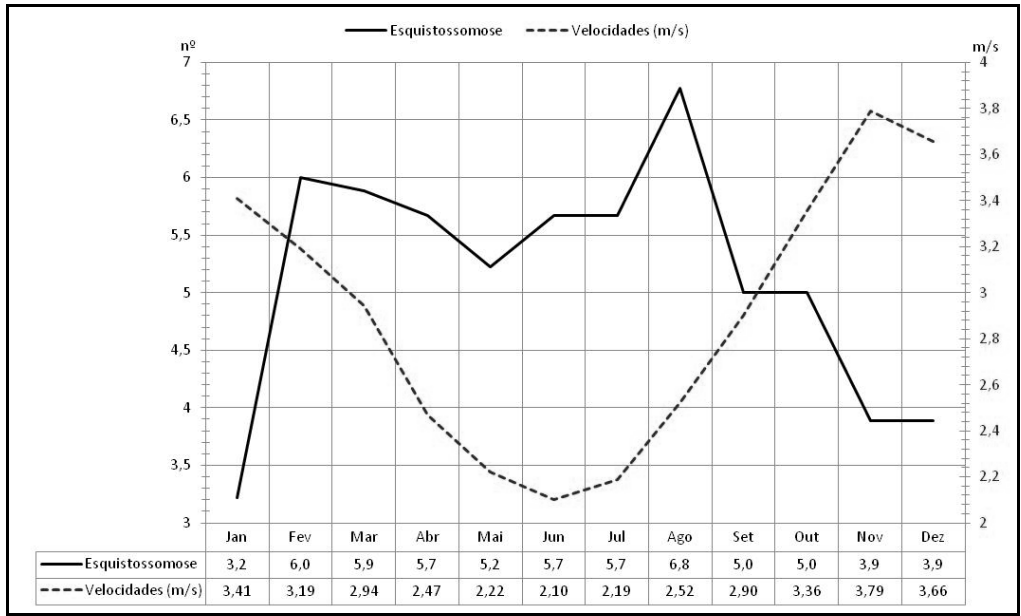

Figura 7 - Relação entre totais mensais dos números de internamentos hospitalares na cidade de Maceió-AL ocasionados por esquistossomose e a média da velocidade do vento. 
vento apresenta período com maiores magnitudes no período de outubro a fevereiro, já no período de maior calmaria se encontra os maiores casos de internamentos pela doença.

Nas relações entre as variáveis meteorológicas e casos de internações (Figura 8) observa-se, que entre as seis variáveis meteorológicas, a insolação e a velocidade do vento possuem uma maior correlação com os números de internações hospitalares por esquistossomose. As outras variáveis estudadas também se destacam por possuírem uma correlação média com a esquistossomose. Mostrando que quanto maior a precipitação pluviométrica, pressão atmosférica, umidade relativa do ar e menor a insolação, temperatura do ar e velocidade do vento, poderá haver mais casos de internações hospitalares por esquistossomose.

O coeficiente de determinação, entre as seis variáveis meteorológicas (Figura 9), mostra que a insolação e a velocidade do ar possuem os melhores resultados com aproximadamente $45 \%$ de relação com os casos de internações hospitalares por esquistossomose.

O caso de Insuficiência Cardíaca (Figura 10) possui inúmeras variações durante o ano,.destacando-se os meses de fevereiro, junho, agosto e novembro, por possuírem os maiores picos da doença, e os meses de janeiro, abril, julho, outubro e dezembro os maiores picos negativos da doença. Relacionando a temperatura do ar ao número de internamentos na cidade de Maceió, constatasse uma relação inversa pouco significativa ao decorrer do ano, entre a linha que representam o número de casos de internações e a linha que representa a temperatura do ar. A temperatura do ar apresenta suas maiores magnitudes entre os meses de novembro a maio. Os meses de agosto registram elevados registros de internações (134,8 casos), notadamente um dos meses com menores valores de temperatura do $\operatorname{ar}\left(23^{\circ} \mathrm{C}\right)$.

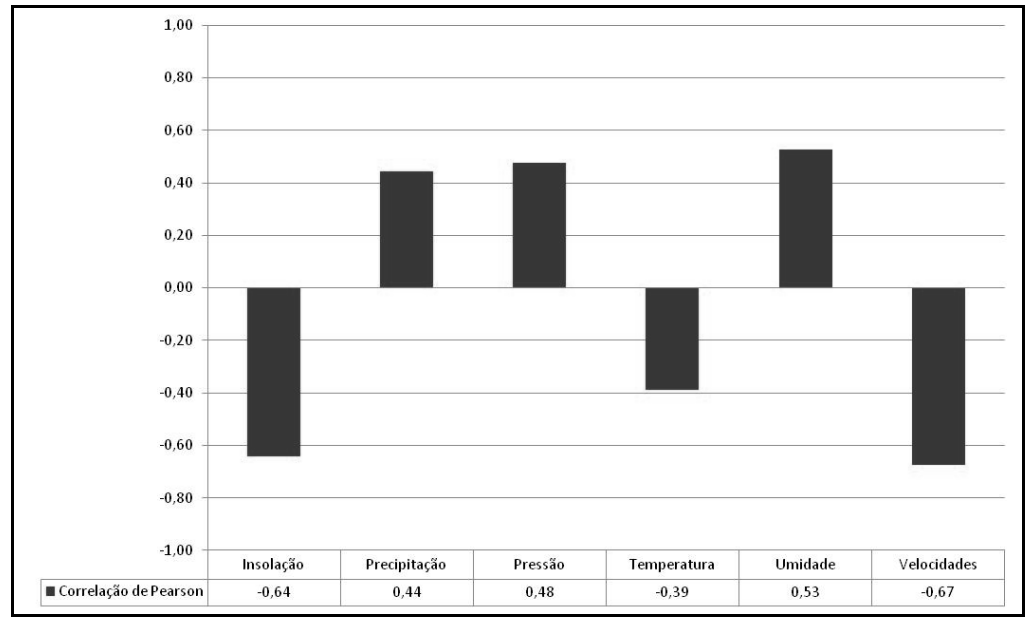

Figura 8 - Correlação de Pearson entre totais mensais dos números de internamentos hospitalares na cidade de Maceió-AL ocasionados por esquistossomose e a média de seis variáveis meteorológicas.

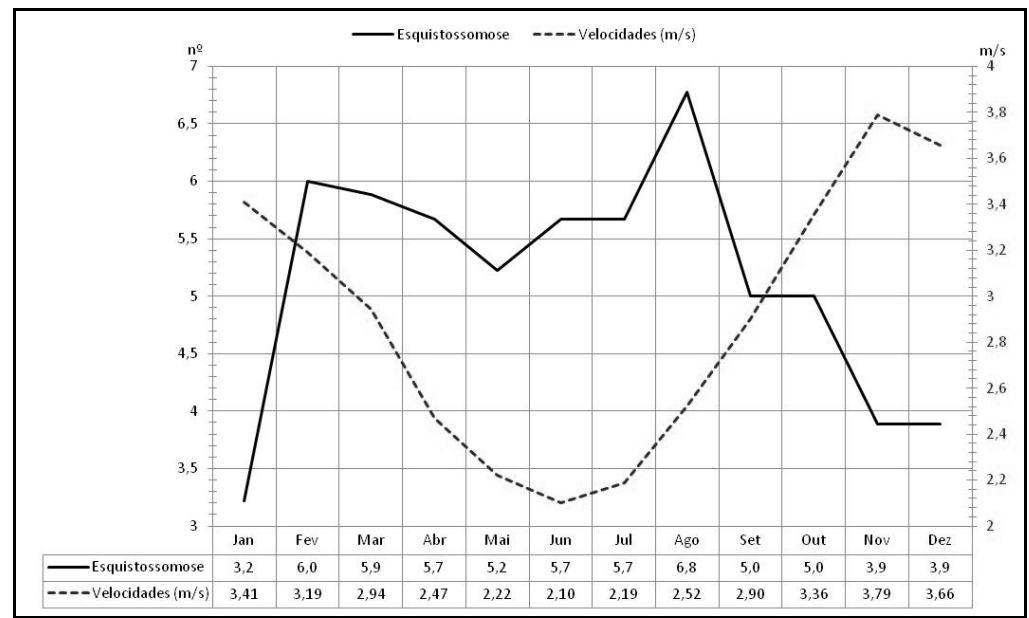

Figura 9 - Coeficiente de Determinação entre totais mensais dos números de internamentos hospitalares na cidade de Maceió-AL ocasionados por esquistossomose e a média de seis variáveis meteorológicas. 
Analisando as relações entre as variáveis meteorológicas e os casos de internações nota-se na Figura 11, que entre as seis variáveis meteorológicas, a pressão atmosférica e a temperatura do ar possuem uma maior correlação com os números de internações hospitalares por insuficiência cardíaca. As outras variáveis estudadas, também, não possuem uma correlação significativa com a insuficiência cardíaca. Portanto, pode-se deduzir que quanto maior a pressão atmosférica e menor a temperatura do ar, poderá haver mais casos de internações hospitalares pela doença estudada.

Através da Figura 12 nota-se que o coeficiente de determinação entre as seis variáveis meteorológicas mostra que a pressão atmosférica e temperatura do ar possuem $11 \%$ de relação com os casos de internamento.

Os valores da leptospirose possuem uma grande elevação no mês de junho, durando até o mês de outubro (Figura 13).
Destacando-se os meses de julho, agosto de setembro por possuírem mais casos de internação (11,1, 11,6 e 13 casos), e os meses de janeiro e novembro por terem menos (1,4 e 1,9 casos). Analisando a pressão atmosférica do ar e os casos de números de internamentos na cidade de Maceió, observa-se que possuem uma relação positiva. A linha representativa da pressão atmosférica distribui-se de forma regular durante o ano, com uma grande elevação entre os meses de maio e baixa nos meses de outubro. É notada uma relação forte entre a leptospirose e a pressão atmosférica, onde os 3 meses de maior número de internamento são os meses de maiores valores de pressão atmosférica.

A relação entre as variáveis meteorológicas e os casos de internações (Figura 14) mostra, que entre as seis variáveis meteorológicas, a pressão atmosférica, insolação, temperatura do ar e umidade relativa do ar possuem uma correlação maior com os números de internações hospitalares por leptospirose. As

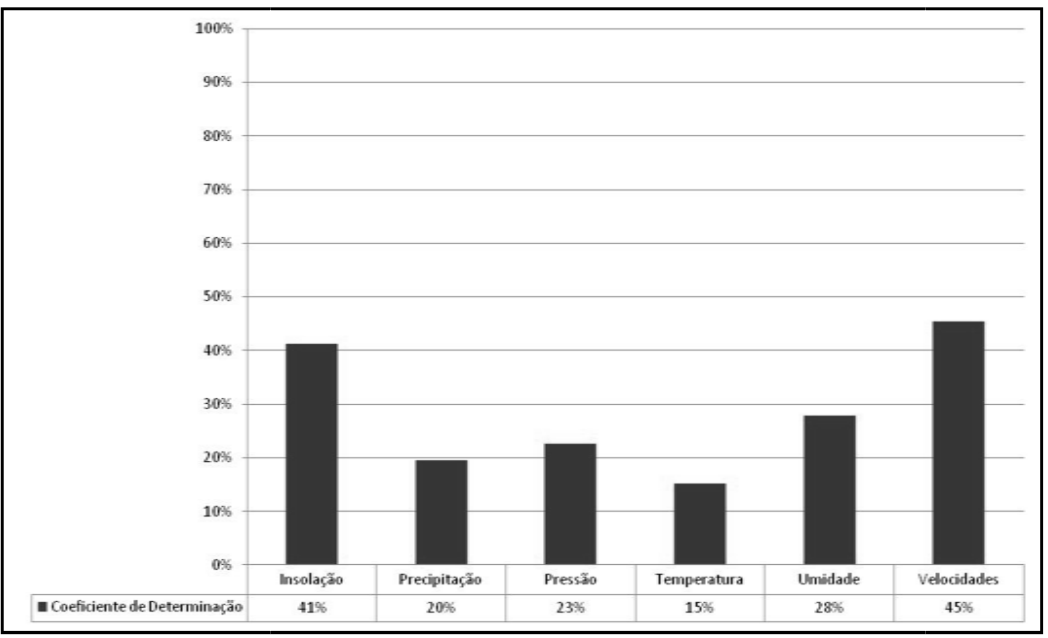

Figura 10 - Relação entre totais mensais dos números de internamentos hospitalares na cidade de Maceió-AL ocasionados por insuficiência cardíaca e média da temperatura do ar.

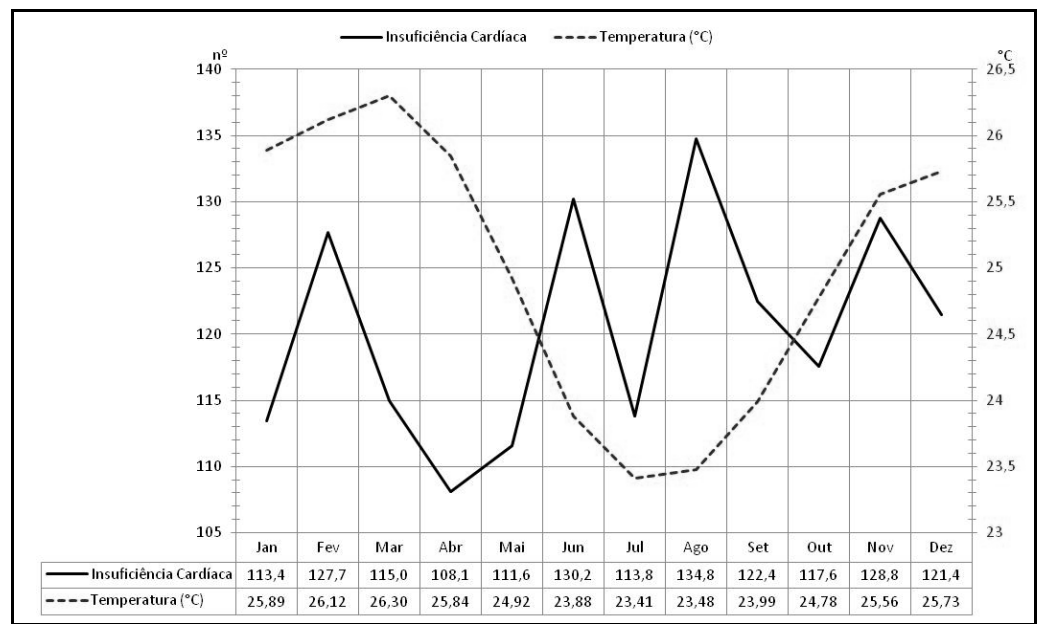

Figura 11 - Correlação de Pearson entre totais mensais dos números de internamentos hospitalares na cidade de Maceió-AL ocasionados por insuficiência cardíaca e média de seis variáveis meteorológicas. 


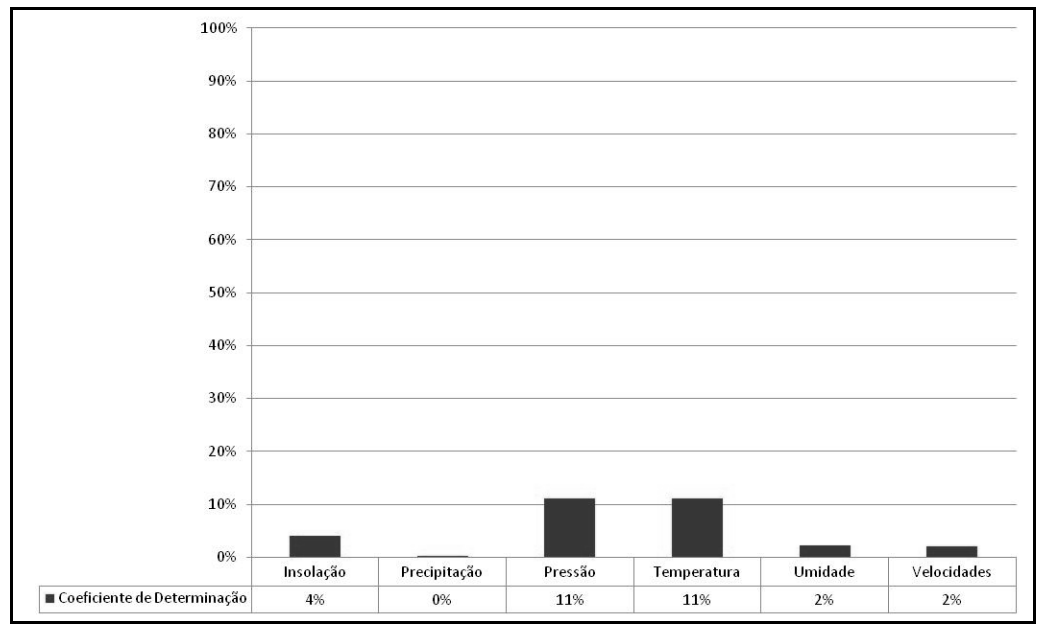

Figura 12 - Coeficiente de Determinação entre totais mensais dos números de internamentos hospitalares na cidade de Maceió-AL ocasionados por insuficiência cardíaca e média de seis variáveis meteorológicas.

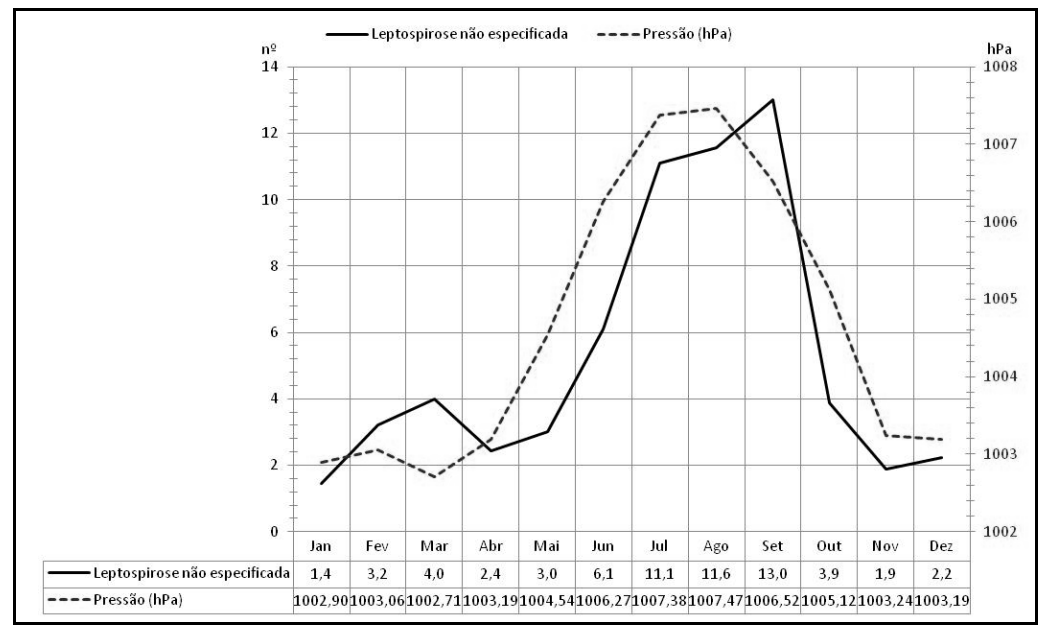

Figura 13- Variação entre totais mensais dos números de internamentos hospitalares na cidade de Maceió-AL ocasionados por leptospirose e média da pressão atmosférica.

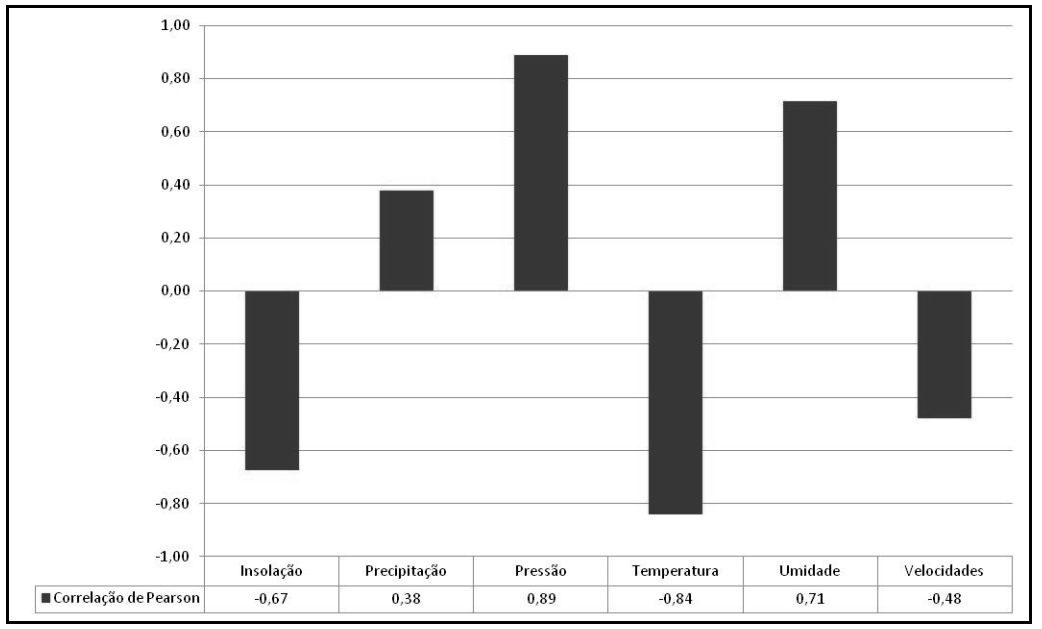

Figura 14 - Correlação de Pearson entre totais mensais dos números de internamentos hospitalares na cidade de Maceió-AL ocasionados por leptospirose e média de seis variáveis meteorológicas. 


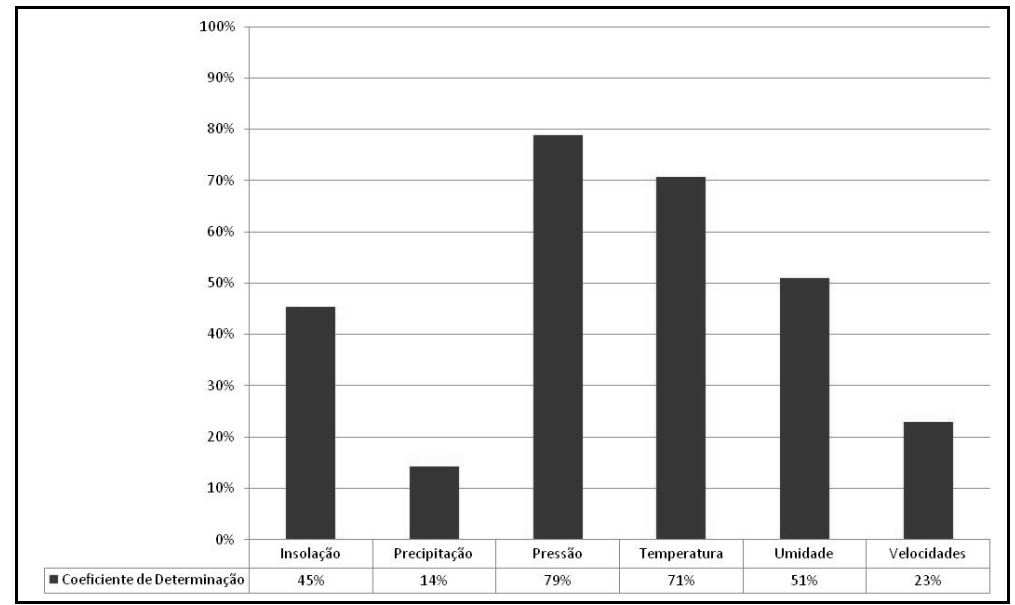

Figura 15 - Coeficiente de Determinação entre totais mensais dos números de internamentos hospitalares na cidade de Maceió-AL ocasionados por leptospirose e média de seis variáveis meteorológicas.

outras variáveis estudadas também se destacam por possuírem uma correlação média com a leptospirose. Mostrando que quanto maior a precipitação pluviométrica, pressão atmosférica e umidade relativa do ar, e menor insolação, temperatura do ar e velocidade do vento, poderá haver mais casos de internações hospitalares por leptospirose.

Foi observado na Figura 15, que o coeficiente de determinação entre as seis variáveis meteorológicas, a pressão atmosférica e a temperatura do ar são as variáveis que possuem os melhores resultados com aproximadamente $79 \%$ e $71 \%$ de relação com os casos de internações hospitalares por leptospirose.

\section{CONCLUSÕES}

Todas seis variáveis estudadas mostraram ter influência em algumas doenças estudadas, mesmo as variáveis que não são utilizadas com frequência nesta área.

Algumas das doenças estudadas obtiveram correlações inferiores à classificação "Média" com as variáveis meteorológicas (asma e a insuficiência cardíaca), mostrando que essas doenças estudadas possuem uma maior relação com outros fatores não meteorológicos. A asma obteve, segundo o coeficiente de determinação, aproximadamente 9\% (Correlação fraca) de influência pela pressão atmosférica e a temperatura do ar. A insuficiência cardíaca foi influenciada também por estas duas variáveis, mais com aproximadamente 11\% (Correlação fraca) de influência.

Algumas doenças se mostraram ser bastante influenciáveis por variáveis meteorológicas, sugerindo que se tenha mais ênfase a estudos desta área. Dentre as variáveis estudadas a insolação apresentou 45\% (Correlação média) de influência sobre a esquistossomose, em relação à bronquite aguda a velocidade do vento, a precipitação pluviométrica e a umidade relativa do ar influenciaram em 47\%, 55\% e 53\% (Correlações médias) dos casos de influência nos casos delas.

O destaque nos resultados foi da Leptospirose com até $79 \%$ (Correlação forte) de correlação com a pressão atmosférica e com a temperatura com cerca de $71 \%$ (Correlação forte) de correlação, mostrando que estas duas variáveis possuem grande influência sobre esta doença.

\section{BIBLIOGRAFIA}

BETTENCOURT, A. R. C., et al. Educação de pacientes com asma: atuação do enfermeiro. Jornal de Pneumologia, 2002, v. 28, n.4, p.193-200.

BIBLIOMED, E.D. Asma. 2008. Disponível em: http:// boasaude.uol.com.br/lib. Acesso em: 19 de setembro de 2009.

BRASIL. Portal Brasil. 2013. Disponível em: http://www. brasil.gov.br/sobre/saude/

atendimento. Acesso em: 15 de junho de 2013.

CAMPOS, C. Queda na pressão atmosférica relacionada a aumento no número de ataques cardíacos. 2005. Disponível em: http://www.drashirleydecampos.com.br/ noticias/16297. Acesso em: 18 de novembro de 2009.

CAVAlCANTE, J. C. Mortalidade em menores de um ano: utilização de novos indicadores para avaliação. Dissertação de mestrado em Saúde da criança, Centro de Ciências da Saúde. Departamento de Tocoginecologia. Universidade Federal de Alagoas. 2003.

DATASUS. MINISTÉRIO DA SAÚDE. Disponível em: www. datasus.gov.br. Acesso em: 12 de agosto de 2009.

EYE, G.V. Insuficiência Cardíaca. 2001. Disponível em: http:// www.abcdasaude.com.br/artigo.php?265. Acesso em: 10 de outubro de 2009. 
LANÇA, M. A. Asma. 2001. Disponível em: http://www. abcdasaude.com.br/artigo.php?38

Acesso em: 10 de outubro de 2009.

LANÇA, M. A. Bronquite Aguda. 2001. Disponível em: http:// www.abcdasaude.com.br/artigo. Acesso em: 13 de outubro de 2009.

OLIVEIRA, E. Leptospirose. 2001. Disponível em: http:// www.abcdasaude.com.br/artigo. Acesso em: 7 de outubro de 2009.

OLIVEIRA, J. C. F. de. Efeitos diretos e indiretos de variáveis meteorológicas na saúde e qualidade de vida da população urbana da cidade de Maceió, AL. Dissertação de mestrado apresentada ao Programa de Pós-graduação em Desenvolvimento e Meio Ambiente-PRODEMA da Universidade Federal da Paraíba-UFPB, para obtenção do grau de mestre. João Pessoa, PB, 2004.
SHIMAKURA, S.E. Coeficiente de determinação. 2006. Disponível em: http://leg.ufpr.br/ silvia/. Acesso em: 27 de outubro de 2009.

TORELLY, A. P. Esquistossomose. 2004. Disponível em: http://www.abcdasaude.com.br/artigo. Acesso em: 15 de novembro de 2009.

UHEREK, E. Condições meteorológicas. 2005. Disponível em: http://www.atmosphere.mpg.de/enid/mais/3 Biometeorologia_4z3.html. Acesso em: 12 de agosto de 2009. 